\title{
Sleep Disturbance in Perimenopausal and Postmenopausal Women: A Cause of Misery
}

Menopause, a natural and physiological occurrence, is not a disease. Importance is always given to reproductive health of female from menarche to menopause. There is an increase in life expectancy with the advent of modern medicine; thus, many women are likely to live for more than 2 decades beyond menopause, in an estrogen deficient state. ${ }^{1}$ Some of the menopausal symptoms experienced by these women can be severe enough to affect the normal lifestyle. Sleep disturbance is a common and important complaint of perimenopausal and postmenopausal women. ${ }^{2}$

The prevalence of difficulties in sleeping is twice as high among middle-aged women as compared with middle-aged men. ${ }^{3}$ Leger et $\mathrm{al}^{5}$ reported sleeping problems in $78 \%$ of women. The prevalence of sleep disturbance is $16 \%$ to $42 \%$ during premenopause, $39 \%$ to $47 \%$ during perimenopause and $35 \%$ to $60 \%$ during postmenopause. 1 Prevalence of insomnia is reported by $25 \%$ of women and severe insomnia by $15 \%$ among women over age 50.5 Sleep problems are significantly worse in surgically postmenopausal women than in naturally occurring postmenopausal women. ${ }^{4}$

Sleep abnormalities may include initiating and/or maintaining sleep with frequent nocturnal and early morning awakenings, problems in waking up, daytime sleepiness and fatigue.3 Difficulties in sleeping may be related to estrogen withdrawal during menopause, which slows the metabolism of serotonin, and serotonin metabolism has an essential role in regulating sleep.3Moreover, some problems commonly encountered during menopause, such as hot flushes and sweating at night, can cause women to have difficulty in sleeping. ${ }^{3}$ Other causative factors that may play a role in sleep disturbances include decreasing reproductive hormone levels, circadian rhythm abnormalities, coexistent medical conditions, primary insomnia, medication, mood disorders, and lifestyle factors. ${ }^{2}$

Sleep quality is an important determinant of health and quality of life. ${ }^{4}$ Chronic insomnia leads to poor work performance, drowsiness, fatigue-related accidents, memory and cognitive impairment. ${ }^{5}$ Sleep problem may also be the first sign of depression. ${ }^{5}$ Long-term health implication of sleep deprivation include high blood pressure stroke and diabetes. ${ }^{6}$ Sleep disturbance associated with menopause is a risk factor for impaired daytime functioning, development of medical and affective disorders, and increased health care costs it may negatively affect the health and safety of postmenopausal women and people with whom they interact. ${ }^{7}$

Recognition of specific conditions or causative factors of sleep disturbance should be identified rather than treating these women nonspecifically with sedative or hypnotics as these drugs have addictive potential. ${ }^{7}$ The Women's Health Initiative study influenced many postmenopausal women to discontinue hormone replacement therapy forcing them and their health care providers to find alternative to estrogen. The only nonhormonal treatment licensed in the United States for moderate vasomotor symptoms is paroxetine $7.5 \mathrm{mg}$. It significantly increases the duration of sleep at night and reduces the nighttime awakenings without sedation.7 A number of complementary and alternative therapies are becoming popular among women seeking alternative to estrogen for management of postmenopausal symptoms. Insufficient data are available to recommend any complementary and alternative therapy as an effective treatment for sleep disturbances associated with menopause. ${ }^{8}$

In conclusion, sleep disorders can lead to impairment of quality of life including psychosomatic, social, and physical activities. In the periodic assessments of menopausal women, it is necessary to concentrate on diagnosis and treatment of sleep disorders. In addition, evaluation of effectiveness of different treatment methods is necessary in this group. Further research is recommended in this aspect of menopausal health, especially in South Asian countries.

\section{REFERENCES}

1. Singh A, Pradhan SK. Menopausal symptoms of postmenopausal women in a rural community of Delhi, India: A cross-sectional study. J Midlife Health 2014 Apr;5(2):62-67.

2. Moreno-Frias C, Figueroa-Vega N, Malacara JM. Relationship of sleep alterations with perimenopausal and postmenopausal symptoms. Menopause 2014 Sep;21(9):1017-1022.

3. Timur S, Sahin NH. Effects of sleep disturbance on the quality of life of Turkish menopausal women: a population-based study. Maturitas 2009 Nov 20;64(3):177-181.

4. Ameratunga D, Goldin J, Hickey M. Sleep disturbance in menopause. Intern Med J 2012 Jul;42(7):742-747.

5. Polo-Kantola P, Erkkola R. Sleep and the menopause. J Br Menopause Soc 2004 Dec;10(4):145-150.

6. Yazdi Z, Sadeghniiat-Haghighi K, Ziaee A, Khadijeh E, Ziaeeha M. Influence of sleep disturbances on quality of life of Iranian menopausal women. Hindawi Psychiatr J 2013;2013:5.

7. Pinkerton JV, Joffe H, Kazempour K, Mekonnen H, Bhaskar S, Lippman J. Low dose paroxetine (7.5 mg) improves sleep in women with vasomotor symptoms associated with menopause. Menopause 2014 Aug 18. [Epub ahead of print].

8. Nedrow A, Miller J, Walker M, Nygren P, Huffman LH, Nelson HD. Complementary and alternative therapies for the management of menopause-related symptoms: a systematic evidence review. Arch Intern Med 2006 Jul 24;166(14):1453-1465. 